\title{
Implementation of Improved Gaussian Filter Algorithm for Retinal Fundus Images
}

\author{
S.A. Jameel \\ Assistant Professor \\ P.G and Research Department of \\ ComputerScience, Jamal Mohamed College \\ (Autonomous) \\ Tiruchirappalli 620 020, Tamil Nadu, India
}

\author{
A.R. Mohamed Shanavas \\ Associate Professor \\ P. G and Research Department of Computer \\ Science, Jamal Mohamed College (Autonomous) \\ Tiruchirappalli 620 020, Tamil Nadu, India
}

\begin{abstract}
Gaussian filter has an important role in edge detection. In this paper, a new methodology for edge detection using Gaussian second order derivative filters is proposed. Channel separation for Retinal Colour Fundus image is used followed by Gaussian filter. The performance of our proposed method has been assessed on 23 images representing the publicly available dataset; High-Resolution Fundus (HRF) Image Database.
\end{abstract}

\section{Keywords}

Gaussian filter, Gaussian second order derivative filter, Fundus image

\section{INTRODUCTION}

The Gaussian filter is broadly used in image processing and computer vision for long years. Generally it works with low pass filtering. [1] Any way for directive filtering a plane cosine wave can be multiplied to the Gaussian function. [2] Its first and second derivatives are also widely used. Among various smoothing filters, the most widely used smoothing filters are Gaussian filters. These filters have been shown to play an important role in edge detection in the human visual system, and to be largely useful as detectors for edge and line detection. Marr and Hildreth displayed that the Gaussian filter (as well as with the Laplacian operator) is same to the difference of Gaussians (DOG) filter. This is a well-known estimation to the shape of spatial amenable fields in the visual system of cats that has also been proposed for humans. Babaud et al verified that when one-dimensional (1-D) signals are softened with a Gaussian filter, the scale space characterization of their second derivatives exhibits that current zero-crossings disappear when moving from a fine-tocoarse measurement, but new ones are not designed. They also proved that for a broad category of signals, the Gaussian function is the unique filter that has this characteristic. This special characteristic makes it possible to track zero-crossings over a range of scales, and also delivers the capability to reclaim the whole signal at sufficiently small scales. Yuille and Poggio enhanced this work to two-dimensional (2-D) signals and demonstrated that with the Laplacian, the Gaussian function is the only filter in extensive category that does not produce zero-crossings as the scale increases. They also showed that for non-sequential directional derivatives along the gradual slope, there is no filter that does not produce zero-crossings as the scale rises.[3] Another important characteristic of the Gaussian filter is the only operator which satisfies the inconstancy relation

$\Delta x \Delta \omega \geq \frac{1}{2}$
Where $\Delta \mathrm{x}$ and $\Delta \mathrm{w}$ are its variance in spatial and frequency domains, correspondingly. This property allows the Gaussian operator to produce the finest tradeoff between the conflicting objectives of the restriction in spatial and frequency domains at the same time. The 2-D Gaussian filter is also the only rotationally uniform filter that is distinguishable in Cartesian coordinates. Separability is significant for computational efficiency when executing the smoothing operation by convolutions in the spatial domain. [1] Marr and Hildreth also declared that the best smoothing filter for images should be confined in both spatial and frequency domains, thereby satisfying the uncertainty relation shown in equation (1). Examine the Gaussian operator in two dimensions shown below

$$
g(x, y)=\frac{1}{2 \pi \sigma^{2}} e^{-\left(x^{2}+y^{2} / 2 \sigma^{2}\right)}
$$

Where $\mu=$ mean, with $\mu=0, \sigma^{2}=$ variance, $\sigma$ is the standard deviation of the Gaussian function and (x,y) are the Cartesian coordinates of the image. Marr and Hildreth suggested that by applying Gaussian filters of different scales $(\sigma)$ to an image; a set of images with different measures of smoothness can be acquired. To find the edges in these images it is necessary to detect the zero-crossings of their second derivatives. The 2-D Gaussian filter is mostly implemented as an image preprocessing step for image softening and noise reduction. The second derivative of Gaussian filter shown in equation (3) is the simple supplement of the Gaussian first derivative filter (equation 2). This can be applied independently to each dimension. Marr and Hildreth accomplished this by applying the Laplacian of a Gaussian (LOG) function as a filter. [1]

$$
\begin{aligned}
& \Delta^{2} g(x, y)=\frac{d^{2}}{d x^{2}} g(x, y)+\frac{d^{2}}{d y^{2}} g(x, y) \\
& =\frac{x^{2}+y^{2}-2 \sigma^{2}}{2 \pi \sigma^{6}} e^{-\left(x^{2}+y^{2} / 2 \sigma^{2}\right)}
\end{aligned}
$$

A technique for saliency estimation established on an image concept into structurally representative elements and contrastbased saliency measures, which can be consistently formulated as high dimensional Gaussian filters was presented. [4] A novel approach for achieving high-quality edge-preserving filtering of images and videos in real time was presented. [5] A flexible scheme for accelerating spatially varying high-dimensional Gaussian filters. [6] An adaptive Gaussian filter algorithm was proposed. [7] A parameterized 
logarithmic image processing (PLIP) method based on Laplacian of Gaussian filtering to improve lung swelling in chest radiographs was proposed. [8]

\section{PROPOSED GAUSSIAN SECOND ORDER DERIVATIVE FILTERS METHOD}

\subsection{Preprocessing}

In the preprocessing stage, the blood vessels in the given fundus image are enhanced as they have non-uniform illumination. The green channel of the fundus image has best vascular structure. Hence, this channel is only considered for enhancement before blood vessel extraction. The first one is channel separation in the processing step. The retinal colour (RGB) fundus image in figure 1 composes of three channels: red, green, and blue channel. [9]

\subsection{Gaussian $2^{\text {nd }}$ order derivative filter}

The blood vessels in the fundus images are enhanced by applying Gaussian $2^{\text {nd }}$ order derivative filter as shown in figure 2 successively by varying sigma. In order to obtain the enhanced blood vessels, the maximum frequency response is chosen from the responses of applied Gaussian filters. The flowchart of the proposed algorithm is shown in figure 3. The proposed algorithm for Gaussian Second Order Derivative Filters is twofold.

Step 1: Design of Gaussian filter array in $x x, x y$ and $y y$ direction

Step 2: Apply Gaussian filter blood vessel detection

\subsection{Algorithm}

Design of Gaussian filter array in $\mathrm{xx}, \mathrm{xy}$ and yy direction

$$
\begin{aligned}
& \operatorname{Gaussians}(\mathrm{x}, \mathrm{x})=\left(X^{2} / \sigma^{2}-1\right) / 2 \pi \sigma^{4} * \\
& e^{-\left(X^{2}+Y^{2} / 2 \sigma^{2}\right)} \\
& \text { Gaussians(x, y) }=(X Y), 2 \pi \sigma^{6} * e^{-\left(X^{2}+Y^{2} / 2 \sigma^{2}\right)} \\
& \operatorname{Gaussians}(\mathrm{y}, \mathrm{y})=\operatorname{Gaussians}(\mathrm{x}, \mathrm{x}) \text { ' } \\
& \text { Apply Gaussian filter blood vessel detection } \\
& \mathrm{D}(\mathrm{x}, \mathrm{y})=\operatorname{Conv}(\mathrm{G}, \operatorname{Gaussians}(\mathrm{x}, \mathrm{x})) \\
& \mathrm{D}(\mathrm{x}, \mathrm{y})=\operatorname{Conv}(\mathrm{G}, \operatorname{Gaussians}(\mathrm{x}, \mathrm{y})) \\
& \mathrm{D}(\mathrm{y}, \mathrm{y})=\operatorname{Conv}(\mathrm{G}, \operatorname{Gaussians}(\mathrm{y}, \mathrm{y})) \\
& \mathrm{D}(\mathrm{x}, \mathrm{x})=\operatorname{multiply}\left(\mathrm{D}(\mathrm{x}, \mathrm{x}), \sigma^{2}\right) \\
& \mathrm{D}(\mathrm{x}, \mathrm{y})=\operatorname{multiply}\left(\mathrm{D}(\mathrm{x}, \mathrm{y}), \sigma^{2}\right) \\
& \mathrm{D}(\mathrm{y}, \mathrm{y})=\text { multiply }\left(\mathrm{D}(\mathrm{y}, \mathrm{y}), \sigma^{2}\right)
\end{aligned}
$$

\section{EXPERIMENTAL RESULTS}

For performance assessment, the proposed technique is tested using the publicly available dataset High-Resolution Fundus (HRF) Image Database.[10] The GUI for Proposed Gaussian Second Order Derivative Filters is shown in figure 4.

\subsection{Dataset}

The public database contains at the present fifteen images of healthy patients, fifteen images of patients with diabetic retinopathy. The proposed method uses fifteen images of healthy patients, eight images of patients with diabetic retinopathy out of 15 images.[10]

\section{DISCUSSION}

The proposed method is used to enhance the blood vessels; in our experiments, a set of 23 colour retinal images from the publicly available datasets were used. This gives good opportunity to test the algorithm on images with different features; normal, abnormal, different sizes. The algorithm is implemented using Matlab. For performance assessment, the proposed technique is tested using the publicly available dataset High-Resolution Fundus (HRF) Image Database.

\section{CONCLUSION}

Many works have been proposed for Gaussian Filter in image processing. Specifically in this work a Second order derivative filter is used for retinal fundus images. In this paper, a new algorithm for designing of Gaussian second order derivative filters is proposed. This is achieved by means of preprocessing followed by derivative filters. The maximum frequency response is chosen from the responses of applied Gaussian filters. The proposed algorithm is tested using the publicly available database. In future this paper is extended to implement a novel method for segmenting the blood vessels by thresholding approach.

\section{REFERENCES}

[1] Basu, Mitra. "Gaussian-based edge-detection methods-a survey." IEEE Transactions on Systems, Man, and Cybernetics, Part C 32.3 (2002): 252-260.

[2] Choomchuay, Somsak, and Keokanlaya Sihalath. "An application of second derivative of gaussian filters in fingerprint image enhancement." Bioinformatics and Biomedical Engineering (iCBBE), 2010 4th International Conference on. IEEE, 2010.

[3] Raman Maini (2012) Analysis and development of image edge detection techniques. PhD thesis. Punjabi University. http://hdl.handle.net/10603/3660.

[4] Perazzi, Federico, et al. "Saliency filters: Contrast based filtering for salient region detection." Computer Vision and Pattern Recognition (CVPR), 2012 IEEE Conference on. IEEE, 2012.

[5] P Gastal, Eduardo SL, and Manuel M. Oliveira. "Domaintransform for edge-aware image and video processing." ACM Transactions on Graphics (TOG). Vol. 30. No. 4. ACM, 2011.

[6] Baek, Jongmin, and David E. Jacobs. "Accelerating spatially varying Gaussian filters." ACM Transactions on Graphics (TOG). Vol. 29. No. 6. ACM, 2010.

[7] Deng, G., and L. W. Cahill. "An adaptive Gaussian filter for noise reduction and edge detection." Nuclear Science Symposium and Medical Imaging Conference, 1993., 1993 IEEE Conference Record.. IEEE, 1993.

[8] Bao, Chen, and Chen Sheng. "A parameterized logarithmic image processing method based on Laplacian of Gaussian filtering for lung nodules enhancement in chest radiographs." Instrumentation and Measurement, Sensor Network and Automation (IMSNA), 2013 2nd International Symposium on. IEEE, 2013. 
[9] R. Hashim, Fatma. A., Nancy. M. Salem, and Ahmed Farag Seddik. "Optic disc boundary detection from digital fundus images." Journal of Medical Imaging and Health Informatics 5.1 (2015): 50-56.

[10] Jan Odstrcilik, R. Kolar, A. Budai, J. Hornegger, J. Jan, J. Gazarek, T. Kubena, P. Cernosek, O. Svoboda, and E.
Angelopoulou, "Retinal vessel segmentation by improved matched filtering: Evaluation on a new highresolution fundus image database," IET Image Process., vol. 7, no. 4, pp. 373-383, 2013.

\section{APPENDIX}
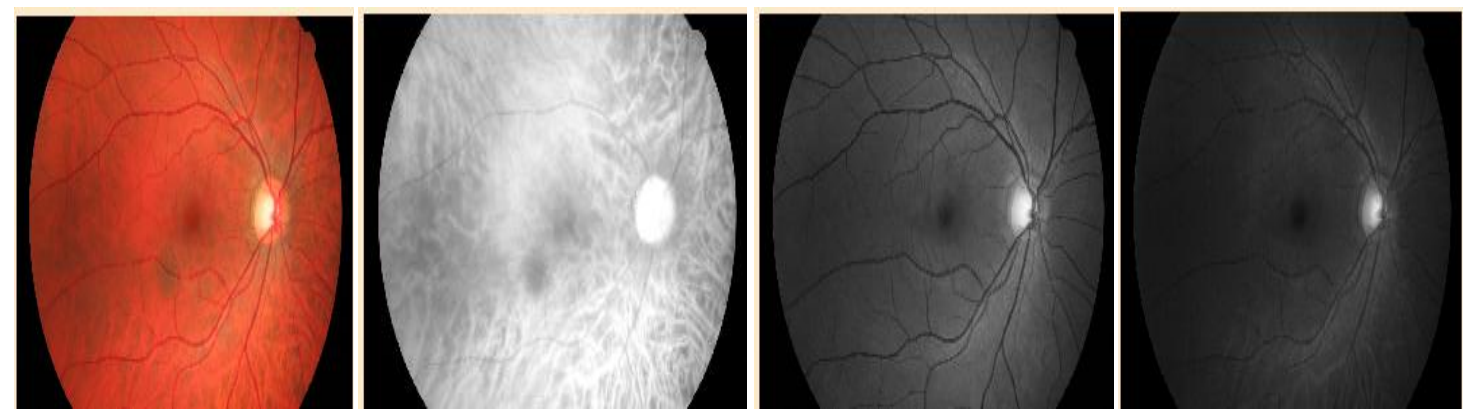

Healthy
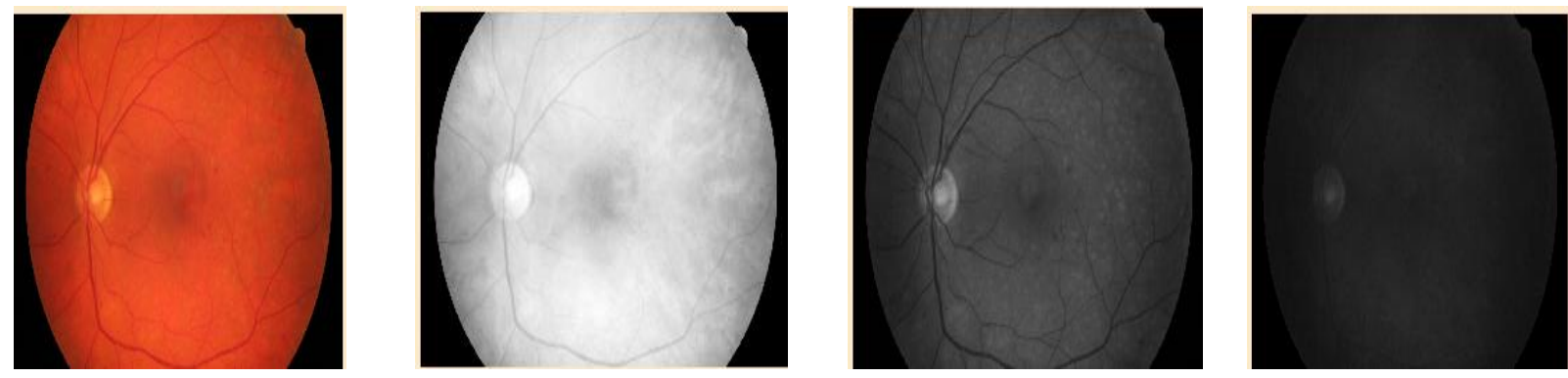

Diabetic Retinopathy
(a) RGB image
(b) Red Channel
(c) Blue Channel
(d) Green Channel

Fig 1: Colour Retinal Images
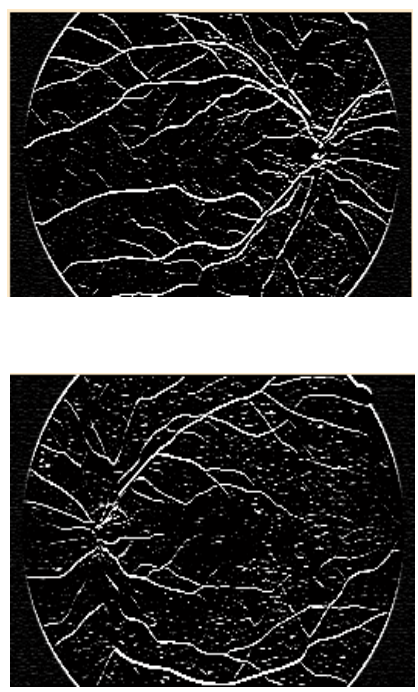

(a) $\mathrm{xx}$-Direction

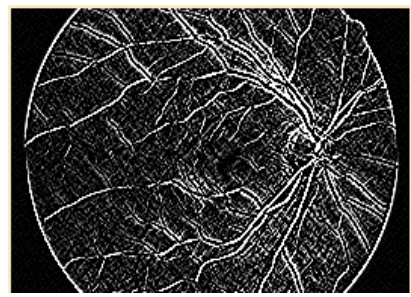

Healthy

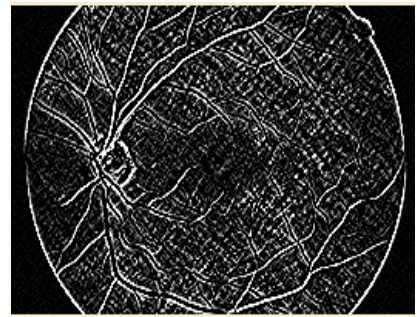

Diabetic Retinopathy

(b) xy-Direction

Fig 2: Derivative Filters

(c) yy-Direction
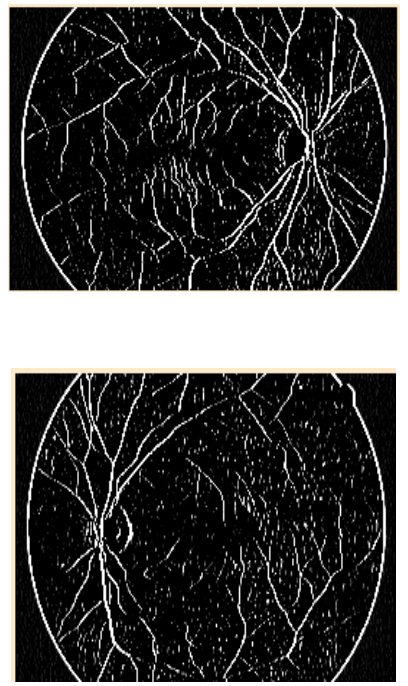


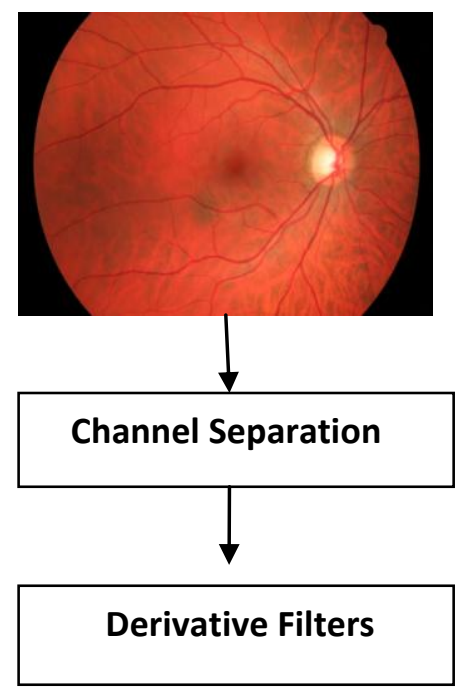

INPUT

\section{PREPROCESSING}

Fig 3: Flowchart of the proposed algorithm

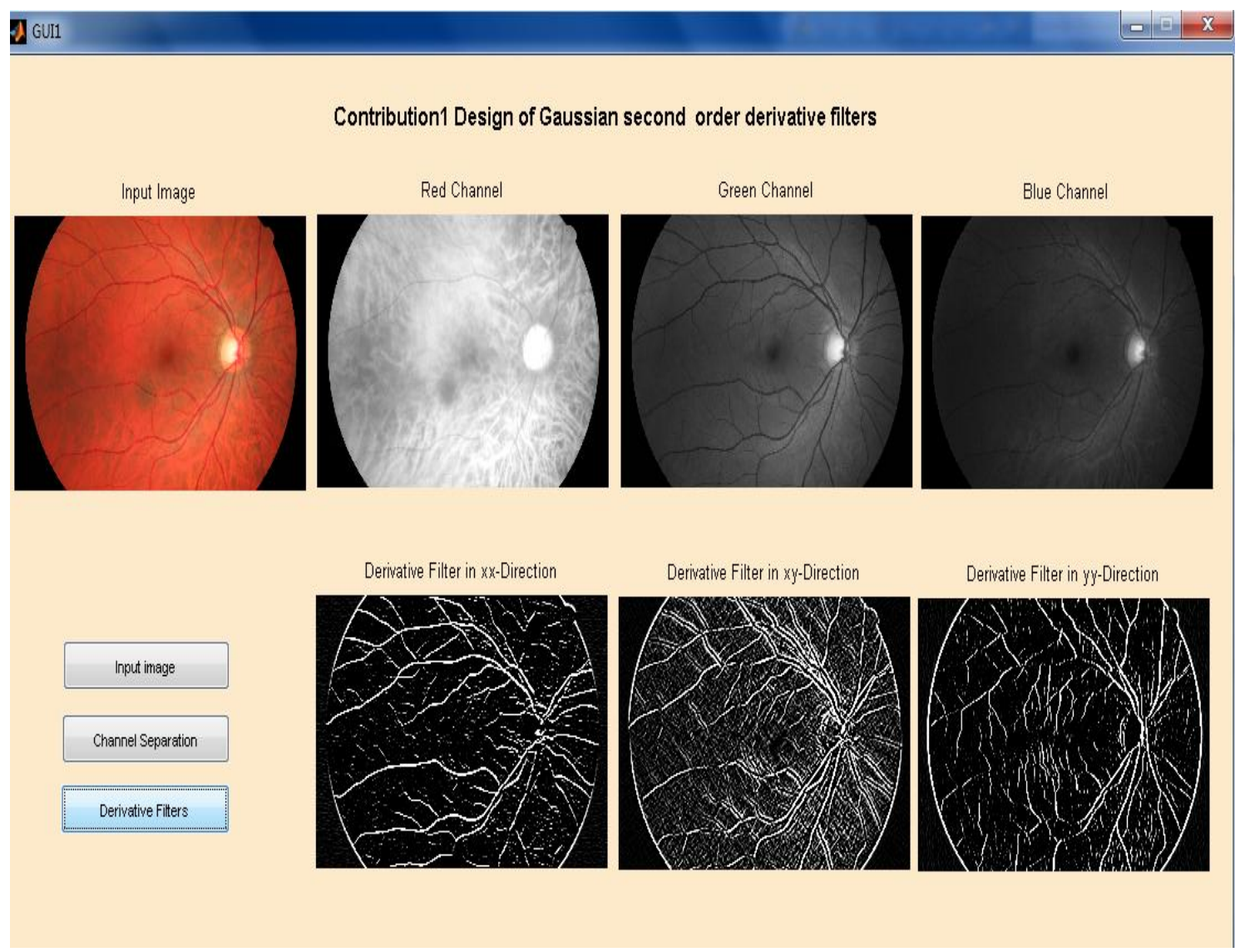

Fig 4: GUI for Proposed Gaussian Second Order Derivative Filters 